\title{
A Control System of SEMG Signal Based on Deep Learning
}

\author{
Weibo Song ${ }^{1,2}$, Wei Wang ${ }^{1}$, Fengiiao Jiang ${ }^{2}$ \\ 1.School of Control Science and Engineering, Dalian University of Technology, Dalian, China \\ 2.College of Information Engineering, Dalian Ocean University, Dalian, China \\ Received: April 24, 2020. Revised: May 29, 2020. Accepted: June 25, 2020. Published: July 21, 2020.
}

\begin{abstract}
The research of control system based on SEMG signal is a popular field at present. It collects bioelectricity of human body through surface electrode. It has the new characteristic of subject fusion, and it is the combination of engineering technology and medical theory, specifically the application of cross combination of control science and electrophysiology. In this paper, the human surface EMG signal is taken as the research object, and a manipulator control system based on one-dimensional convolutional neural network $(\mathrm{CNN})$ is proposed, and the functions and implementation methods of each part of the system are analyzed. The experimental results show that the recognition accuracy of the training model is 0.973 , and the design scheme of EMG signal recognition and classification system with deep learning method is feasible. The successful design of the system provides technical support and theoretical basis for the further study of electrophysiological signals.
\end{abstract}

Keywords-Deep Learning, EMG Signal, Neural Network, Electrophysiological Technology.

\section{INTRODUCTION}

$\mathrm{I}_{\mathrm{e}}^{\mathrm{n}}$ $n$ recent years, both researchers in the field of control engineering and doctors in the field of electrophysiology have paid great attention to the processing and recognition of EMG signals, which has become a research hotspot, thus promoting a new situation of cooperation between medical and engineering departments. This cooperation has made the research and application of EMG signals develop rapidly.

Through the body's muscle activity and nervous system adjustment, and use of electrophysiological technology to detect and obtain EMG signals, especially the introduction of artificial intelligence and the application of big data, it rapidly promoted the new way of intelligent medical treatment and auxiliary diagnosis, so the research of EMG signal control system based on deep learning is very meaningful, and provides

This work was supported by Scientific Research Fund Project of The Educational Department of Liaoning Province(No.QL201913; No.QL2017017; No.QL2017018); Natural Science Fund of Department of Science and Technology of Liaoning Province (No.201602101). Thanks to my tutor and electrophysiological expert team of Dalian Medical University for helping me finish the experiment. guidance for the control of intelligent prosthesis. So as to improve the recognition rate of correct gesture and the portability of system function.

Literature $[1,2]$ studies the acquisition and preprocessing of signals, literature $[3,4]$ studies the recognition and classification of signals, and has achieved some results, but there are still some problems in the above literature, including the accuracy of continuous multi actions recognition, and the content of the research is discrete, so this paper proposes the integration of the current popular deep learning technology, designs and realizes a complete set The whole EMG signal control system. In this paper, only convolutional neural network method is used to classify gestures [5-7], and the classification results are matched to the motors which control the degree of freedom of the manipulator. The introduction of convolutional neural network reduces the time and labor cost of signal feature extraction [8-11].

In this paper, the main purpose of the experiments using the above theory is to realize the feasibility of the system, that is, to verify the following aspects: 1 . Collecting and acquiring EMG signals from the human skin surface; 2. Analyzing and processing these acquired signals; 3 . Classifying different kinds of signals in order to form instructions for controlling the manipulator installed on the robot. The previous experiments are to threshold the acquired signals to get the required level signal. It is also a trigger signal, which is to be processed and analyzed by Arduino hardware platform. It is programmed on this platform to monitor the trigger level that conforms to a certain gesture. When the level signal is recognized, the manipulator will perform a series of actions [12-15]. The structure of the paper is as follows: the second part after the foreword is the overview of system structure design; the third part discusses the key technologies of the system; the fourth part is the experimental verification of the system; and then the summary and Acknowledgment part.

\section{SySTEM ARCHITECTURE OVERVIEW}

The control system of robot based on sEMG signal is composed of sEMG signal acquisition equipment, computer, wireless transceiver (Bluetooth), 4-DOF robotic arm and its 
control circuit, force / visual feedback control unit. The characteristics of the robot control system is to obtain the sEMG signal through non-invasive surface electrode, which can continuously recognize and classify different gestures. Due to the integration of deep learning technology, the accuracy of system identification is high, misoperation is reduced, and operation efficiency is improved.

The signal acquisition device is worn on the operator's arm to collect the EMG signals of the corresponding part, and the collected signals are front-end processed (such as the signal amplification and filtering process), and then the signals processed by the front-end are sent to the computer for the next signal core processing. This process is to complete the identification and classification of different movement gestures of the operator.

The classification results are converted into the control commands of the manipulator motor rotation, and transmitted to the main control system of the manipulator through wireless mode and distributed to each motor of the manipulator. At the same time, a force tactile / visual monitoring system is set up to feedback the grasping information of the manipulator end to the human brain for auxiliary adjustment of the control amount. Thus a complete set of closed-loop feedback system is realized, which is an effective way to improve the control accuracy[16]. The structure of the manipulator control system based on the human surface EMG signal is shown in Fig 1.

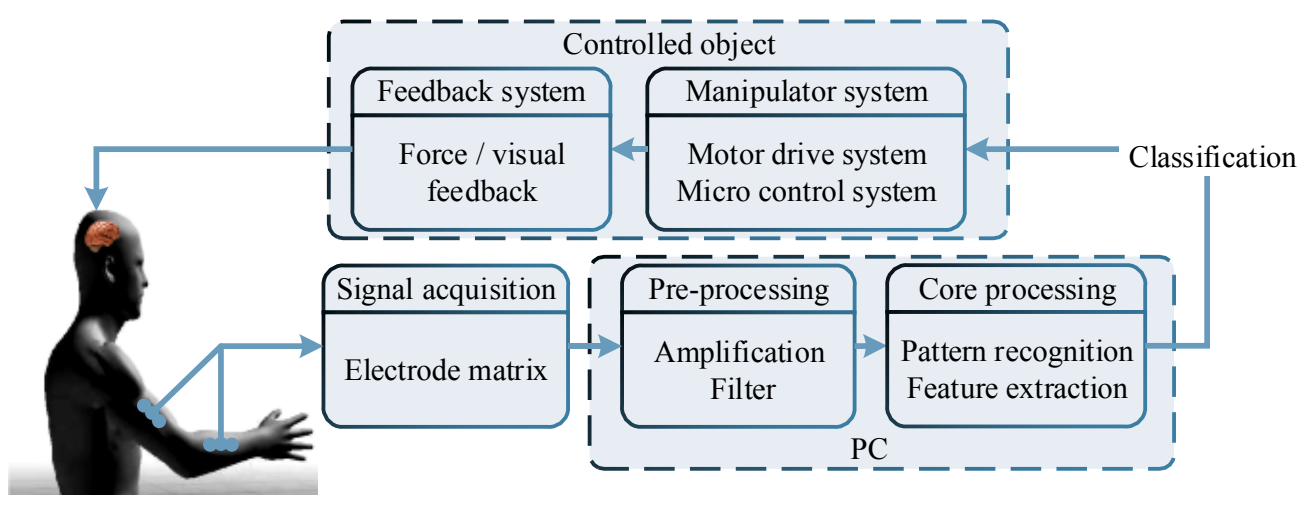

Fig. 1. Structure of manipulator control system based on sEMG signal

\section{KEY TECHNOLOGY}

This part mainly introduces the main functions and implementation methods of each part of the manipulator control system based on the electric signal of human surface muscle. This process involves many key technologies.

\section{A. Signal acquisition}

The acquisition part of the signals is the front-end unit of the manipulator control system based on the electromyography of the human body. This process is very important for the quality of the collected human surface signal. Including: The first problem is selection of electrodes in contact with human skin and the arrangement of electrode array. In consideration of the safety of the operator, the surface electrode which is not damaged to the skin is selected in the experimental process, while the invasive electrode is abandoned which is not necessary in this system, it can be applied in the examination of some muscle diseases to get more accurate signals in the muscle. The second problem is the location of the electrodes, which should be considered when the muscle contraction changes obviously during the specific action of the arm, as well as the direction along the muscle extension. Also need to pay attention to the details: in order to prevent large displacement between the electrode wristband and the skin, the position should be marked with a pen after wearing to the correct position for observation and comparison.

\section{B. Signal Pre-processing}

Because the EMG signal collected from the human body surface has obvious characteristics of weak and poor anti-interference ability, the weak is reflected in the amplitude range of the signal is about $10 \mathrm{uv} \sim 5 \mathrm{mv}$, and the frequency range is about $10 \mathrm{~Hz} \sim 500 \mathrm{~Hz}[17]$. Because of the above characteristics of the signals, further processing of the original signal is needed to do the follow-up research, such as identification and classification process. Therefore, after the front-end signal acquisition process, we must use the amplification and filtering process in this part. One of our concerns is how to choose the multiple of amplification. The key point is to enlarge the weak amplitude signal to the range suitable for the system operation, so as to ensure that the signal is not distorted. Another problem is to filter out the interference waves, such as flattening the interference waves in the resting state or filtering out the singular points that may have wrong judgment on the classification results without affecting the normal useful signals. 


\section{Signal Core Processing}

(a)For the original EMG signal generated by a gesture or simple preprocessing in the early stage, Elman neural network $[18,19]$ is used to process it to detect the amplitude of the signal (see Formula 1,Fig 2). The meaning of the parameter is the traditional variable description of the neural network. The parameter $S$ is not reflected in the formula and is cancelled after equal substitution.

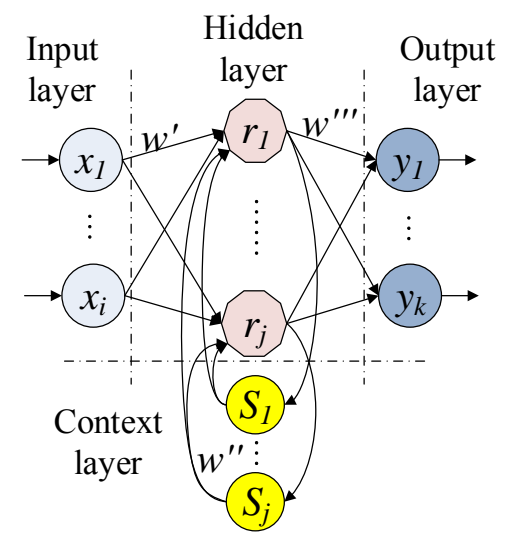

Fig. 2. Elman neural network structure

$$
y(k)=F\left(w^{\prime \prime \prime} H\left(w^{\prime} r(k-1)+w^{\prime \prime} x(k-1)\right)\right)
$$

When the same continuous action, such as relaxation, clenching and other signals are generated. As shown in Fig 3, the continuous action signal is characterized by the obvious increase of amplitude and the alternation of signal intervals with small amplitude. The time span of such interval signal is determined by the duration of gesture action or relaxation action, which is a linear relationship. The signal in such time interval shall be processed accordingly, and such signal will be used for the control command of the controlled object and trigger the command, for example, the control mode of a manipulator.

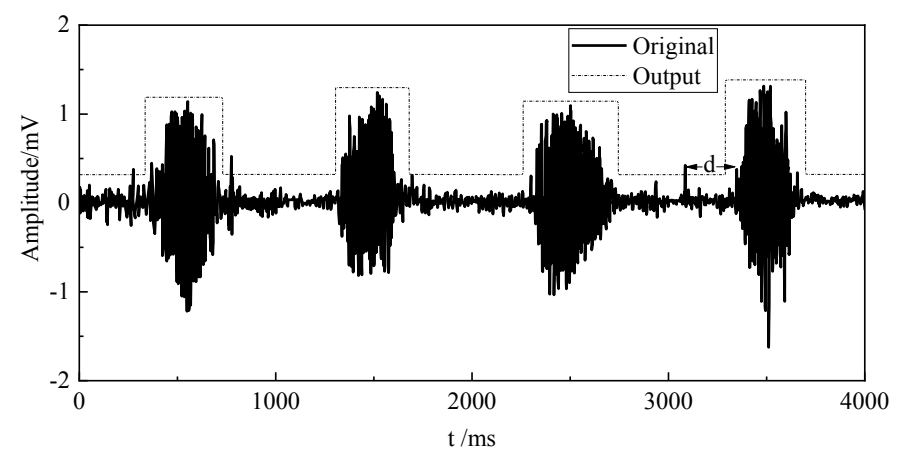

Fig. 3. Continuous signal and output
An important element in such a continuous action is the starting and ending points of the effective signal. It is important to determine the time interval from the starting point to the ending point. Specifically, the control process of the controlled object is not only single time applicable, but also in general is multiple times continuous. In particular, in terms of continuity, it can be understood as a continuous sequence generated by the superposition of a single independent action, with a sequence of resting states between them. In the experiment, the signal amplitude was first obtained from a specific action. After proper filtering to eliminate slight fluctuations, the shaper is processed to output a perfect rectangular pulse (see Fig 3).

The duty cycle $\boldsymbol{Q}$ of this kind of pulse has practical significance (see formula 2). The length of $\boldsymbol{t}$ during the high level can be understood as the effective time length of a certain action, and the selection of the starting point and the ending point of the actions can obtain empirical data through the experimental process. Of course, this process is not a simple discrimination process, and it should be considered, for example, whether the amplitude value of the expected condition is for independent point or interference point, the commonly used method is to set another threshold value $\boldsymbol{H}$, which is used for the time distance $\boldsymbol{d}$ between the point and the point densely reaching the threshold condition as shown in Fig 3. Whether the point is identified as the judgement condition of the starting point or the ending point is shown in Formula 3:

$$
Q=t / T
$$

$$
\boldsymbol{d}=\left\{\begin{array}{l}
<\boldsymbol{H}, \text { Starting and ending condition } \\
\geq \boldsymbol{H}, \text { Not Starting and ending condition }
\end{array}\right.
$$

(b) In the core signal processing, how to recognize different gestures and classify them is the key of the algorithm. In this paper, the gesture classification method based on deep learning is adopted, and the Tensorflow framework system[20-23] launched by Google is used, among which many algorithms can be used. This paper proposes a gesture classification method based on one-dimensional convolutional neural network, and the classification results are matched to the motors which control the degree of freedom of the manipulator. The integration of $\mathrm{CNN}$ reduces the time and labor cost of signal feature extraction. The model architecture of $\mathrm{CNN}$ is shown in Fig 4. The process uses 8-way electrode array as the input, after convolution and pooling processing, the convolution kernel size is $3 \times 3$, the pooling kernel is $2 \times 2$, and the ReLu activation function is selected. After the expansion process, the final step is to the full connection layer step to achieve classification output [24-27]. See Fig 5 for the way to match the output results to the manipulator. After training, verification, testing and other necessary processes, an 
algorithm model suitable for the recognition and classification of sEMG signals is obtained. The model algorithm can be extended to practical commercial applications.

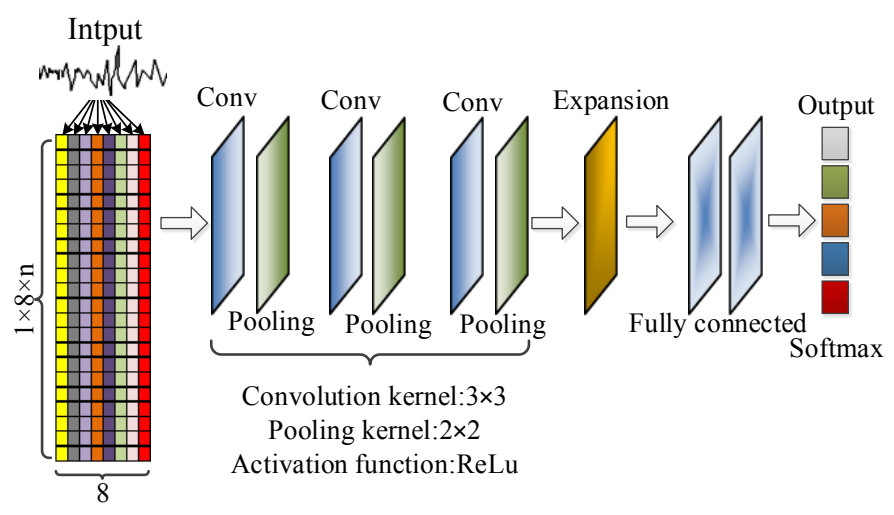

Fig. 4. Convolution neural network structure

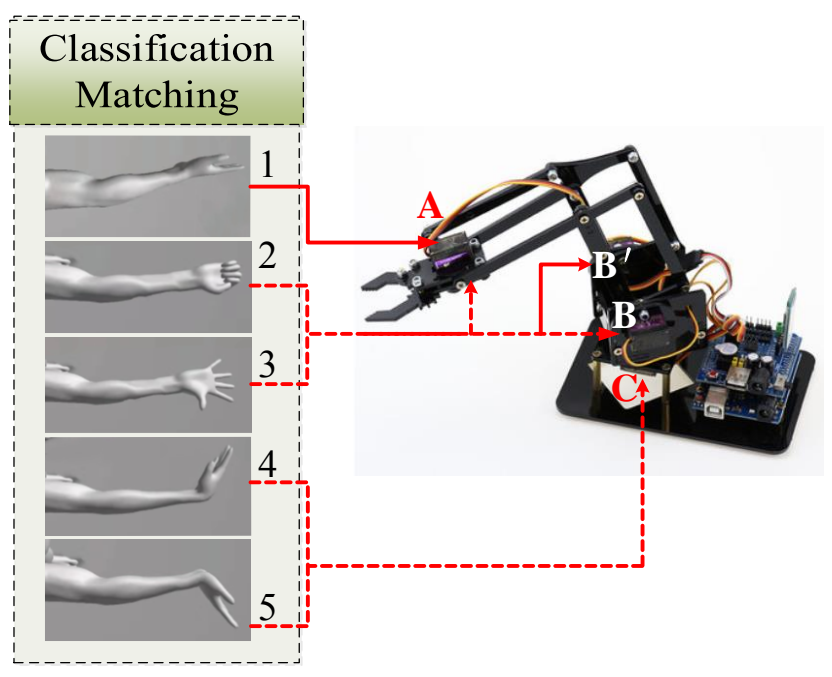

Fig. 5. Classification results matching

The control process of the manipulator is actually to define the processed EMG signal as the trigger level, which includes: high level will trigger the motor to rotate; low level will stop or reset. According to the sequence of program execution, the specific matching process includes: 1. Gesture interface definition function gesturedef(), which is used to detect predefined gestures and obtain trigger high level, so as to trigger motor rotation control; 2. Motor rotation control function moveto(); 3. Rotation function turnround(), including: rotation angle, rotation time and other parameters. During the operation of the system, the movement of the manipulator and the movement of the hand with wrist strap meet the real-time requirements. According to the operation of the manipulator, real-time adjust the gesture movement to obtain the control amount, and finally achieve the closed-loop control[28,29].

\section{EXPERIMENT}

After theoretical description, this part is verified by experiments. The wristband with 8 pairs of electrodes is worn to the best position of the arm (see Fig 6), which is used to effectively identify arm movements and gestures. If the wristband is not placed properly, it will affect the quality of the collected surface EMG signal and affect the process of signal recognition and classification, reduce the accuracy of these processes, and eventually lead to the poor stability and practical application of the control system. Correctly wear the wristband in the area with more muscle groups on the lower arm to ensure that the electrode of the wristband can correctly recognize muscle movement. In order to meet the experimental requirements, the selected robot system includes Arduino system control board, 8-way motor drive control board, 4-DOF robotic arm, wireless transmission module (Bluetooth Technology).

The experimental processes are mainly for gesture recognition and classification, some details are not shown. A complete set of experimental control processes are required to go through the following steps: 1 . The end of the manipulator starts from point $\mathrm{A}, 2$. The end of the manipulator is moved to point $\mathrm{B}$ through the hand outward action, 3 . The manipulator grabs the object through the clenching action, 4 . The hand inward action moves the end of the manipulator to point $\mathrm{C}$, and 5. The hand stretching action releases the object. The detailed processes are shown in Fig 6. 

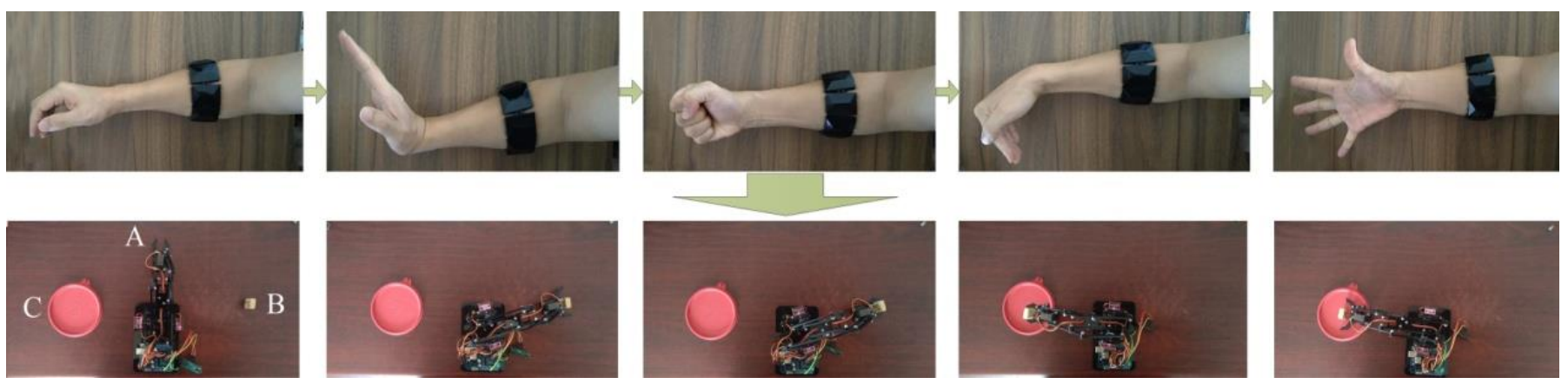

Fig. 6. Experimental processes

In this paper, one-dimensional convolution neural network is used to train the collected sEMG signals to obtain gesture classification model. The source of the data set is obtained by collecting the sEMG signals of the operator. The total data of each gesture contains 200 complete gesture motion states, of which 100 are used for training, 60 are used for validating and 40 are used for testing. When the training rounds are 500, the change of accuracy is in a stable state and the fluctuation is small, so it is used as the optimization and stopping criterion point of model. The accuracy curve is shown in Fig 7. At this time, the recognition accuracy of the training model is 0.973 .

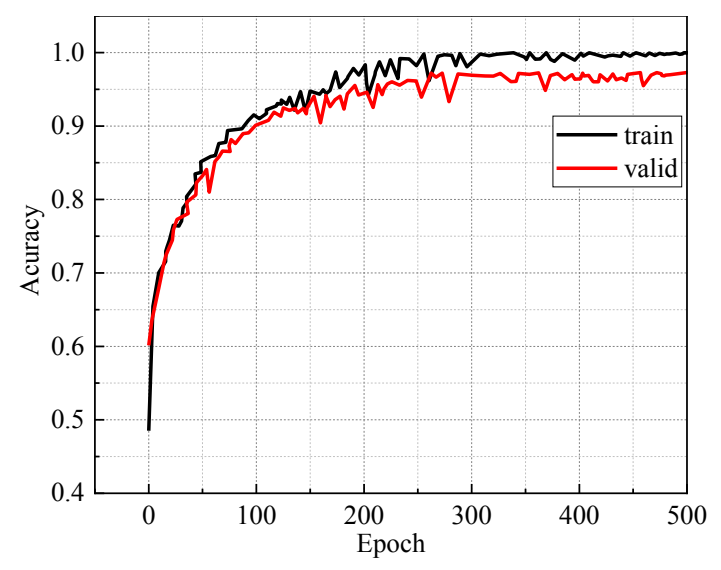

Fig. 7.Accuracy curve

The above model is used to control the manipulator. Each gesture controls the manipulator movement 100 times respectively. The number of times that the manipulator can correctly follow the motion intention of each gesture and complete the operation task are shown in TABLE I. The results show that the correct recognition rate of each gesture is more than $96 \%$. Compared with the results of other similar literatures, it is found that the correct recognition rate of this method is at a high level, so the performance of the system to a certain extent meets the needs of sEMG signals recognition and classification.
TABLE I. Success rate of gesture control

\begin{tabular}{cc}
\hline Gesture classification & Success rate (\%) \\
\hline Rest state & 100 \\
Hand outward & 96 \\
Clench fist & 97 \\
Hand inward & 97 \\
Palm expansion & 96 \\
\hline
\end{tabular}

At the same time, the system also has some limitations. For example, due to the limitations of the mechanical structure of the manipulator, the control flexibility is limited; because of the operator's repeated gesture movement for a long time, it is easy to entangle the hand fatigue accumulation phenomenon, which makes the collected signal affected and reduces the recognition rate.

\section{V.CONCLUSIONS}

For the above experiments, the manipulator can follow the gesture to complete the intention of the operator, and achieve the grasping control task of the manipulator. The design and debugging of the control system has successfully realized a path from input to output. In order to quickly realize the system and its functions, the relative basic methods are used in each link of the process. The system proposed in this paper has a strong learning ability and can achieve efficient feature extraction. In order to maintain a high correct recognition rate, operators need to have some experience to ensure that the sEMG signal of each gesture is stable and specific. If the intelligent prosthesis or the intelligent control process is realized, the manipulator can be replaced by the prosthesis of the humanoid arm, and the classification signal can be matched to the motor corresponding to the degree of freedom, and the rotation amount and rotation speed can be controlled. 
Therefore, the next work will be the in-depth improvement of theory and algorithm. So as to improve the recognition rate of correct gesture and the portability of system function.

\section{REFERENCES}

[1] Hoadley K D , Warner M E. Use of Open Source Hardware and Software Platforms to Quantify Spectrally Dependent Differences in Photochemical Efficiency and Functional Absorption Cross Section within the Dinoflagellate Symbiodinium spp[J]. Frontiers in Marine Science, 2017, 4,365-.

[2] Li X , Samuel O W, Zhang X, et al. A motion-classification strategy based on sEMG-EEG signal combination for upper-limb amputees[J]. Journal of NeuroEngineering and Rehabilitation, 2017, 14(1):1-13.

[3] Khoshdel, Vahab, et al. sEMG-based impedance control for lower-limb rehabilitation robot[J]. Intelligent Service Robotics, 2018, 11.1: 97-108.

[4] Waris, Asim, et al. Multiday evaluation of techniques for EMG-based classification of hand motions. IEEE journal of biomedical and health informatics, 2018, 23.4: 1526-1534.

[5] Alba-flores, Rocio, et al. Performance analysis of two ANN based classifiers for EMG signals to identify hand motions. IEEE SoutheastCon, 2016, 1-5.

[6] Kehri, Vikram, et al. EMG Signal Analysis for Diagnosis of Muscular Dystrophy Using Wavelet Transform, SVM and ANN[J]. Biomedical and Pharmacology Journal, 2018, 11.3: 1583-1591.

[7] Tsinganos, Panagiotis, et al. Deep Learning in EMG-based Gesture Recognition[J]. PhyCS. 2018, 107-114.

[8] Chung, Edison A, et al. Real-Time Hand Gesture Recognition Model Using Deep Learning Techniques and EMG Signals[J]. 27th European Signal Processing Conference (EUSIPCO). IEEE, 2019, 1-5.

[9] Nodera, Hiroyuki, et al. Deep learning for waveform identification of resting needle electromyography signals[J]. Clinical Neurophysiology, 2019, 130.5: 617-623.

[10] Alaskar, Haya Mohammad Abdulaziz. Deep Learning of EMG Time-Frequency Representations for Identifying Normal and Aggressive Actions. International Journal of Computer Science and Network Security, 2018, 18,12.16-25.

[11] Calderon-Cordova, Carlos, et al. EMG signal patterns recognition based on feedforward artificial neural network applied to robotic prosthesis myoelectric control. Future Technologies Conference (FTC). IEEE, 2016, 868-875.

[12] Oleinikov, Artemiy, et al. Feature extraction and real-time recognition of hand motion intentions from EMGs via artificial neural networks. 6th International Conference on Brain-Computer Interface (BCI). IEEE, 2018, 1-5.

[13] Gandolla, Marta, et al. Artificial neural network EMG classifier for functional hand grasp movements prediction. Journal of International Medical Research, 2017, 45.6: 1831-1847.

[14] Park K H, Lee S W. Movement intention decoding based on deep learning for multiuser myoelectric interfaces[C], 4th International Winter Conference on Brain-Computer Interface (BCI). IEEE, 2016, 1-2.
[15] Zia ur Rehman M, Waris A, Gilani S O, et al. Multiday EMG-based classification of hand motions with deep learning techniques[J]. Sensors, 2018, 18(8): 2497.

[16] Samadani A. Gated recurrent neural networks for emg-based hand gesture classification. a comparative study[C].40th Annual International Conference of the IEEE Engineering in Medicine and Biology Society (EMBC). IEEE, 2018: 1-4.

[17] Mao Chen, et al. Design of sEMG signal acquisition system [J]. Electronic technology, 2017, 46 (11): 62-64.

[18] Kolanowski, et al. Multisensor data fusion using Elman neural networks. Applied Mathematics and Computation, 2018, 319: 236-244.

[19] Simão M, Neto P, Gibaru O. EMG-based online classification of gestures with recurrent neural networks[J]. Pattern Recognition Letters, 2019, 128: 45-51.

[20] Li Yaoqing. Research and application of convolutional neural network in pathological diagnosis [D]. Shenzhen University, 2018.

[21] Qi Zhaohui. Application of convolution neural network based on tensorflow [D]. Wuhan University, 2018.

[22] Abadi, Martín, et al. Tensorflow: A system for large-scale machine learning. The 12th Symposium on Operating Systems Design and Implementation. 2016, 265-283.

[23] Ameri, Ali. EMG-based wrist gesture recognition using a convolutional neural network. Tehran University Medical Journal TUMS Publications, 2019, 77.7: 434-439.

[24] Soman S, et al. Improved sEMG signal classification using the Twin SVM[C]//2016 IEEE International Conference on Systems, Man, and Cybernetics (SMC). IEEE, 2016: 004507-004512.

[25] Simon A M , Hargrove L J , Lock B A, et al. The Target Achievement Control Test: Evaluating real-time myoelectric pattern recognition control of a multifunctional upper-limb prosthesis[J]. Journal of Rehabilitation Research \& Development, 2011, 48(6):619.

[26] Hasni H, Yahya N, Asirvadam V S, et al. Analysis of Electromyogram (EMG) for Detection of Neuromuscular Disorders[C] International Conference on Intelligent and Advanced System (ICIAS). IEEE,2018: 1-6.

[27] Al Mashhadany Y I, Awsaj M K. Detection of muscles diseases based on EMG signal by using locally recurrent neural networks (LRNNs) techniques[J]. International Journal of Engineering \& Technology, 2018, 7(4): 4850-4855.

[28] Faust, O. , Hagiwara, Y., Hong, T. J. , Lih, O. S. , \& Acharya, U. R. Deep learning for healthcare applications based on physiological signals: a review. Computer Methods \& Programs in Biomedicine, 2018, 161: 1-13.

[29] Chambon, S., Galtier, M. N., Arnal, P. J., Wainrib, G., \& Gramfort, A. A deep learning architecture for temporal sleep stage classification using multivariate and multimodal time series. IEEE Transactions on Neural Systems and Rehabilitation Engineering, 2018,26(4), 758-769.

\section{Creative Commons Attribution License 4.0 (Attribution 4.0 International, CC BY 4.0)}

This article is published under the terms of the Creative Commons Attribution License 4.0

https://creativecommons.org/licenses/by/4.0/deed.en_US 